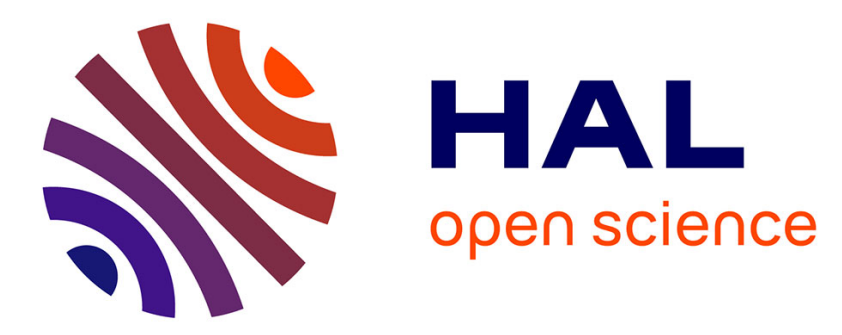

\title{
Development of wireless, batteryfree gyroscope based on one-port SAW delay line and double resonant antenna
}

Chen Fu, Omar Elmazria, Frederic Sarry, Thaiyan Mahalingam, Sang Sik Yang, Keekeun Lee

\section{To cite this version:}

Chen Fu, Omar Elmazria, Frederic Sarry, Thaiyan Mahalingam, Sang Sik Yang, et al.. Development of wireless, batteryfree gyroscope based on one-port SAW delay line and double resonant antenna. Sensors and Actuators A: Physical, 2014, 220, pp.270-280. 10.1016/j.sna.2014.10.006 . hal-01293187

\section{HAL Id: hal-01293187 \\ https://hal.science/hal-01293187}

Submitted on 20 Apr 2020

HAL is a multi-disciplinary open access archive for the deposit and dissemination of scientific research documents, whether they are published or not. The documents may come from teaching and research institutions in France or abroad, or from public or private research centers.
L'archive ouverte pluridisciplinaire HAL, est destinée au dépôt et à la diffusion de documents scientifiques de niveau recherche, publiés ou non, émanant des établissements d'enseignement et de recherche français ou étrangers, des laboratoires publics ou privés. 
Development of wireless, batteryfree gyroscope based on one-port SAW delay line and double resonant antenna

\author{
C. Fu ${ }^{1}$, O. Elmazria ${ }^{2}$, F. Sarry ${ }^{2}$, T. Mahalingam ${ }^{1}$, S. Yang ${ }^{1}$, and K. Lee ${ }^{1, *}$ \\ ${ }^{1}$ Department of Electrical Eng., Ajou University, Suwon 442-749, Korea \\ ${ }^{2}$ Institute Jean Lamour, UMR 7198, Université de Lorraine-CNRS, France
}

*K. Lee, tel: +82-31-2191848; keekeun@ajou.ac.kr 


\begin{abstract}
A wireless, battery-free micro-gyroscope was developed by employing a one-port surface acoustic wave (SAW) reflective delay line, a SAW resonator, and an antenna. Two SAW devices with different center frequencies were simultaneously activated by one antenna with double resonant frequencies. An $80 \mathrm{MHz}$ RF energy from antenna activates a double-port SAW resonator while a $195 \mathrm{MHz}$ RF energy activates a one-port SAW delay line. The fabricated gyroscope showed clear reflection peaks with high signal-to-noise $(\mathrm{S} / \mathrm{N})$ ratios in both the time and frequency domains in measurement system during wireless testing. Upon rotation of the device, large shifts of the reflection peaks were observed owing to a secondary wave interference effect caused by the Coriolis force that depends on the spinning rate. The measured sensitivity and linearity of the developed gyroscope were, respectively, $1.35 \mathrm{deg} /(\mathrm{deg} / \mathrm{s})$ and 0.91, which are promising values for our targeted applications. The temperature, metal mass effect, and vibration/shock effects were also studied and the results are discussed.
\end{abstract}

\title{
KEYWORDS
}

Coriolis force, Double resonant antenna, Microgyrosensor, One-port SAW reflective delay line, Surface acoustic wave (SAW)

\section{Introduction}

Various types of gyroscopes are available which work on different mechanisms. Some gyroscope uses a spinning wheel, and the other utilizes Sagnac or Coriolis force effect. Recently, gyroscopes are employed in many applications such as smart phones, digital camera, automobile stabilization system, smart weapons system, rover in space, etc. The specifications of the gyroscope mainly depend on the types of the applications. For smart missile systems or satellite applications, the gyroscope's bias stability should be up to $10^{-4} \mathrm{deg} / \mathrm{hr}$, but for rate 
applications such as smart phones and handheld electronics, the gyroscope has to detect 100 $\mathrm{deg} / \mathrm{hr}$. Among several different types of gyroscopes, the mechanical gyroscope uses the spinning wheel effect, in which the wheel rotates approximately 24,000 revolutions per minute and the center axis of the spinning wheel will be retained in a fixed position. Even though a main body is tilted from its original position, the center axis of the spinning wheel is retained to enable to detect the tilted extent of the main body. Fiber optic and ring laser gyroscopes use the Sagnac effect. Light wave emitted from a semiconductor laser (typically a wavelength of $800 \sim 1500 \mathrm{~nm})$ is radiated to both directions of a fiber optic and then it will propagate along a long length of optical fiber. At the measurement system consisted of photo detector at the exit, both light waves will be formed a harmonic and constructive phase, making the light bright. However, if the system undergoes an angular velocity, both light beams will not be matched in phase due to Sagnac effect at the output, and thus the intensity will be attenuated at the photo detector. Another type of gyroscope is MEMS gyroscope which uses the Coriolis force effect. This kind of MEMS gyroscope has many issues to be resolved. Most of the currently existing MEMS gyroscopes have a single proof-mass suspended above the substrate; it is free to oscillate in two orthogonal directions. Owing to the suspended mechanical structure, the device is very sensitive to external shock and vibration. In addition, when a resonant vibration is applied on the sense axis from the outside, the oscillating device cannot detect the Coriolis force. To obtain a high sensitivity, the MEMS gyroscope requires a matched resonant frequency between the drive mode and the sense mode. However, during fabrication, inevitable imperfections are generated, resulting in an unmatched resonant frequency. Normally, the resonant frequency matching in both the drive and the sense mode can be achieved by electrical tuning, but this requires additional power and control systems.

To overcome the above issues, we suggest a SAW-based gyroscope. In recent years, SAWbased gyroscopes have received a great deal of attention for use in automobile safety systems, 
image stabilization, body movement monitoring, and smart weapons systems applications because these kinds of SAW gyroscopes can provide unique properties, such as superior inherent shock and vibration robustness, easy resonance frequency matching between the drive and the sense mode, low power consumption, and facile wireless circuit implementation [1-4]. To date, few research groups have reported SAW-based gyroscopes with different designs and structures [5-10]. Varadan et al. reported a 74.2 MHz MEMS-IDT SAW gyroscope [8], and Kurosawa et al. demonstrated the effect of the metal dot array design on the gyroscope performance [9],. Lee et al. presented a micro rate gyroscope based on the SAW gyroscopic effect on ST quartz using a differential dual-delay-line oscillator configuration [10],. However, despite these important reports on SAW gyroscopes, they still suffer from low sensitivity, poor temperature stability, complex transceiver circuitry in the sensor itself, difficulty in implementing a wireless gyroscope, heavy battery installation onto the sensor itself to activate the devices, and so on.

In the present work, we introduce a novel wireless, battery-free SAW-based microgyroscope for use in unmanned aircrafts, smart weapon guidance systems, robots, virtual reality games, and other related applications. This kind of gyrosensor eliminates complex transceiver and interface electronic circuits that require thousands of transistors and battery installation onto the sensor for activation; this gyrosensor also has the advantages of high shock/vibration robustness and high sensitivity in harsh environments (Ref).

\section{Operating principles}

Figure 1 shows a schematic view of the fabricated SAW-based gyrosensor system. The overall sensor system is composed of two SAW devices on a single piezoelectric substrate, an antenna with double resonant frequencies, and a network analyzer as a reader system. The antenna has two resonant frequencies: $80 \mathrm{MHz}$ and $195 \mathrm{MHz}$. When electromagnetic (EM) 
energy is radiated to the sensor through the antenna, two SAW devices, a resonator and a oneport delay line, are simultaneously activated. By using $80 \mathrm{MHz}$ EM energy, the SAW resonator, which is composed of two IDTs and reflectors, is activated, giving rise to a stable standing wave between two interdigital transducers (IDTs). Here, metallic dots are placed on the antinode position of the standing wave and vibrate in the normal direction. Using $195 \mathrm{MHz}$ EM energy, the one-port SAW reflective delay line, which is composed of one IDT and three reflectors, is activated. The SAW propagates toward the three reflectors, is reflected at the shorted grating reflectors, and returns back to the IDT. The SAW energy reflected from the three reflectors is converted to EM energy at the IDTs and sent back to the network analyzer system via EM energy with a $195 \mathrm{MHz}$ center frequency. This one-port SAW reflective delay line makes it possible to implement a wireless, battery-free gyroscope system [11-12]. When the substrate is revolved around the standing wave direction, the Coriolis force is induced by the vibrating metallic dots. The Coriolis force generates a secondary SAW in the direction orthogonal to the propagating standing wave. The Coriolis force is proportional to the mass of the metallic dot $(m)$, the vibrating velocity of the metallic dot $(v)$, and the angular rotation of the substrate $(\Omega): F_{\text {coriolis }}=2 \cdot m \cdot\left(v_{\text {saw }} \times \Omega\right)$. This newly generated secondary SAW interferes with the Rayleigh SAW traveling in the one-port reflective delay line, causing a change in the acoustic velocity of the wave by a strain energy, which leads to a shift in the resonant frequency in the frequency domain and shifts in the positions of the reflection peaks in the time domain. By analyzing the deviations in the resonant frequency and time shifts from the one-port SAW reflective delay line, we can extract the rotation rate of the substrate.

\section{Optimal design considerations}

On a $128^{\circ} \mathrm{YX} \mathrm{LiNbO}_{3}$ substrate, two SAW devices, a resonator and delay line, were designed together. The center frequencies of the resonator and delay line were $80 \mathrm{MHz}$ and 195 
$\mathrm{MHz}$, respectively. The resonator is to generate a stable standing wave and the one-port SAW reflective delay line is for wireless, battery-free activation and measurement of the gyrosensor.

SAW resonator: A two-port SAW resonator with reflectors was employed to form a standing wave [13-14]. Several parameters are involved in the SAW resonator, which includes the center frequency, the number of IDT finger pairs, the IDT aperture length, the cavity length between the two IDTs, the spacing between the IDT and its adjacent reflectors, and the number of metal stripes in the shorted grating reflector. A $128^{\circ} \mathrm{YX} \mathrm{LiNbO}_{3}$ was used as the piezoelectric substrate because it has a high Rayleigh wave velocity and large electromechanical coupling coefficient $\left(\mathrm{K}^{2}\right)$. Coupling of Mode $(\mathrm{COM})$ modeling was employed to find optimal resonator parameters prior to fabrication. One parameter was varied while other parameters were kept constant to find optimal results at COM modeling. Based on COM modeling, the optimum parameters obtained are: a $128^{\circ} \mathrm{YX} \mathrm{LiNbO}_{3}$ substrate, an $80 \mathrm{MHz}$ operating frequency, five IDT electrodes, a $40 \lambda$ aperture length, a $50 \lambda$ cavity between the input and output IDTs, a $5 \lambda / 8$ spacing between IDT and its adjacent shorted grating reflectors, and 250 metal stripes for the shorted grating reflector.

Metallic dots: The metallic dots were placed at the antinodes of the standing wave. The weight of a metallic dot $\mathrm{m}$ was calculated to be $10^{-15} \mathrm{~kg}$ when gold is used for metallic dot material, which is lighter than that of proof mass in MEMS gyroscope. However, our structure has an array structure of metallic dots and hence the total Coriolis force is the cumulative value of all the metallic dots placed at the antinodes of the standing wave. The vibrational velocity of a metallic dot is faster than that of proof mass in MEMS gyroscope, which is an additional advantage of enhancing the Coriolis force. Depositing a thick metal with a high mass density could increase the Coriolis force. However, excess mass loading from the metallic dots disturbs 
by crushing and squeezing the propagation of the standing wave, resulting in a change in the resonance frequency in the resonator. On the basis of our simulation analysis the optimum thickness of gold dots were identified as $\sim 250 \mathrm{~nm}$ and used in the development of the gyroscope.

One-port SAW reflective delay line: In the present work, a one-port delay line configuration is used to realize the possibility of battery-free, wireless measurements. A $195 \mathrm{MHz}$ center frequency was used to prevent an extremely large size difference between the resonator and delay line. To obtain sharp reflection peaks, a high $\mathrm{S} / \mathrm{N}$ ratio, and without any spurious peaks, the following design parameters are considered for the reflective delay line: the type of IDT configuration, the number of IDT finger pairs, the aperture length, the types of reflectors, and the number of metal stripes in the shorted grating reflector. COM modeling was performed to find the optimal parameters. All the above parameters were varied and then simulated. The optimal parameters for a one-port SAW reflective delay line with a $195 \mathrm{MHz}$ center frequency were determined. A single phase unidirectional transducer (SPUDT) configuration was chosen to direct most of SAW energy to the reflector directions. Only a minimum amount of RF energy is radiated to the other direction from SPUDT, which minimize the inherent loss and insertion loss. The number of IDT finger pairs was set to 20 . The aperture length was set to $50 \lambda$ to reduce lateral diffraction from the IDT. To obtain uniform intensities in the reflection peaks, the reflectors close to the IDT possess small aperture lengths and relatively few metal stripes, whereas the reflectors far from the IDT have large apertures and many stripes. Of the three reflectors, the first reflector was used for the reference peak, and the other two were for sensor peaks, which vary with the extent of the Coriolis force.

Secondary wave: When the device is subjected under an angular velocity, the Coriolis force is enacted the vibrating metallic dots with velocity $v$ to generate a secondary wave toward 
orthogonal direction to the vibrating metallic dots. A newly generated wave due to the Coriolis force, which we call the secondary wave, propagates toward the delay line. This newly generated secondary wave interferes with the Rayleigh-type SAW traveling in the one-port reflective delay line, causing a change in velocity and amplitude, resulting in a shift in the resonant frequency. The amplitude of the secondary wave decays during the propagation toward the delay line, so that the spacing between the metallic dot array and the delay line should be short. However, extremely short spacing may induce a large parasitic electromagnetic (EM) feed-through between the delay line and the resonator owing to crosstalk. Thus, a proper distance separation between the metallic dots and the sensor delay line is needed. The frequency of the secondary wave is the same as that of the resonator, and the velocity is known to be $3656 \mathrm{~m} / \mathrm{s}$; therefore, the wavelength is estimated to be $45.7 \mu \mathrm{m}$. To confirm the interference effects from the secondary wave in a SAW delay line, we designed and fabricated the configuration such as Fig. 2. SAW is propagating at the delay line from port 1 to port 2, and at the same time, another SAW interferes the propagating SAW from the vertical side. As the input RF power increases from the vertical side as well as the center frequencies becomes higher, the SAW velocity propagating at the delay line becomes slower, and more downshifted at the center frequency as shown in Fig. 2(c).

\section{COM modeling}

To extract the optimal design parameters, coupling of modes (COM) modeling was performed prior to fabrication. COM modeling should consider an acoustic wave propagating in both directions from the IDT and incorporate the coupling interaction that arises because of the reflection from IDT fingers. The total response was obtained by cascading all the components. We assume that all the waves propagating to the wafer edges were fully absorbed and that there is no boundary reflection. Based on COM parameters in Table 1, the respective 
components of SAW devices, IDT, reflectors, and gaps were modeled.

Table 1. COM parameters

\begin{tabular}{ll}
\hline Parameters & Values \\
\hline \hline Velocity $(\mathrm{m} / \mathrm{s})$ & 3920 \\
$k \cdot \lambda($ reflectivity) & $\mathrm{R}_{\mathrm{m}} \cdot(\mathrm{h} / \lambda)+\mathrm{R}_{\mathrm{e}}$ \\
Electrical effect: $\mathrm{R}_{\mathrm{e}}$ & -0.018 \\
Mechanical effect: $\mathrm{R}_{\mathrm{m}}$ & 0.692 \\
Normalized capacitance $\left(\mathrm{pF} / \mathrm{m}^{2}\right)$ & $4.69 \times 10^{2}$ \\
$\gamma$ attenuation $($ Nepers $/$ wavelength) & $3.1 \times 10^{-4}$ \\
Al sheet resistivity $(\Omega \cdot \mathrm{m})$ & $2.7 \times 10^{-8}$ \\
\hline
\end{tabular}

One-port SAW reflective delay line: As shown in Figure 1, the one-port reflective delay line is composed of an IDT, three reflectors, and three free gaps between reflectors. The corresponding P matrixes were cascaded by acoustic port connections in series. Subsequently, the total electric port admittance $\mathrm{Y}_{11}$ was deduced, and then its reflection coefficient $\mathrm{S}_{11}$ was obtained as

$S_{11}=\frac{1-Z_{i n}^{*} Y_{11}}{1+Z_{i n} Y_{11}}$

Here, $Z_{\text {in }}$ is the IDT external input impedance and the symbol '*' stands for the conjugate. Using the inverse FFT algorithm, the $\mathrm{S}_{11}$ in the time domain was easily obtained.

A one-port SAW reflective delay line with a $195 \mathrm{MHz}$ center frequency was simulated. The finger pairs were 20 and the finger numbers in the reflectors were 3,4 , and 5 , respectively. The simulation results for one-port SAW reflective delay line are shown in Figure 3. The solid line is the $\mathrm{S}_{11}$ response with the external matching circuit in the frequency and time domains. The external matching circuit was crucial for determining the efficient reflective delay line response. A $195 \mathrm{MHz}$ center frequency with a narrow bandwidth was observed, and three reflection peaks with high $\mathrm{S} / \mathrm{N}$ ratios and uniform intensities were also observed through $\mathrm{COM}$ simulation. It is found that externally impedance-matched devices showed better performances. 
Two-port SAW resonator: As shown in Figure 1, the two-port SAW resonator is symmetric and it constitutes two IDTs, two shorted grating reflectors, and the cavity between two IDTs. Their COM equations and $\mathrm{P}$ matrixes can be solved and calculated. The $\mathrm{P}$ matrixes were cascaded through acoustic ports in series and electric ports in parallel. Finally, the two-port resonator response was deduced and its transmission coefficient was calculated as

$$
S_{21}=\frac{-2 Y_{21} \sqrt{\mathrm{G}_{1} \mathrm{G}_{2}}}{\left(\mathrm{G}_{1}+\mathrm{jB} \mathrm{B}_{1}+Y_{11}\right)\left(\mathrm{G}_{2}+\mathrm{jB} \mathrm{B}_{2}+Y_{22}\right)-Y_{21} Y_{12}}
$$

where $\mathrm{G}_{\mathrm{i}}$ and $\mathrm{B}_{\mathrm{i}}(\mathrm{i}=1,2)$ are the real and imaginary part of the external circuit admittance toward IDT $\mathrm{i}$, respectively. The period of the IDTs was $48.8 \mu \mathrm{m}$ so that the central frequency is designed to be $80 \mathrm{MHz}$. The IDT finger number was 5, while the reflector finger number was 250. The gap between the IDT and its neighbor reflector was $30.5 \mu \mathrm{m}$, which is equal to $5 / 8$ times the wavelength. The simulated result in the absence of a metal dot array is shown in Figure 4. Wider gaps between two IDTs are preferred to comprise a bigger metal dot array, which signifies a stronger secondary SAW. However, IDT gaps that are too long result in multimode generation and increase the complexity of the resonator's standing wave. Thus, the optimized 50-wavelength gap between the two IDTs was adopted by referring to the results of COM modeling.

Metallic dot effect: The metal dot array plays a key role in the SAW gyroscope. Generally, a thick and heavy metal is preferred to increase the magnitude of the newly generated secondary wave. However, if the metal is too heavy, the central frequency will be shifted and the occurrence of a bulk wave and material damping will deteriorate the device performance. Thus, finding the optimum thickness of a metallic dot is necessary. Taking into account the metal dot array, the finite element method (FEM) was used to model and analyze the influence of the heavy metal dots. A gold metallic dot array was positioned over the gap center of the resonator. 
The size of each metallic dot was $12.4 \mu \mathrm{m} \times 11.4 \mu \mathrm{m}$. The resonator responses with various thicknesses of metal dot arrays are also shown in Figure 4. It can be observed that the $\sim 250$ nm-thick gold metal dot array is well matched with the $80 \mathrm{MHz}$ central frequency.

\section{Fabrication and testing setup}

Using a COM modeling, optimal device parameters were obtained and the results were predicted in advance prior to fabrication. Using the device parameters, the device was fabricated using CMOS process. On a 4" $128^{\circ} \mathrm{YX} \mathrm{LiNbO}_{3}$ wafer, a $2000 \AA$-thick layer of aluminum was deposited and then patterned for both the resonator and delay line. After etching away the aluminum, $\mathrm{Cr} / \mathrm{Au}$ metallic dots were formed using the lift-off process to increase the mass of the metallic dots (Figure 5). The fabricated device was dicing-sawed to pieces and then connected to an antenna.

Next, two identical, isotropic antennas with two resonant frequencies $(80 \mathrm{MHz}$ and 195 $\mathrm{MHz}$ ) were fabricated to activate two different SAW devices at the same time. The bandwidth of the $80 \mathrm{MHz}$ center frequency was set to be narrow for exquisite standing wave generation, but the delay line bandwidth of the $195 \mathrm{MHz}$ center frequency was somewhat broader. For wireless tests, the sensor was placed on the precision rate table in the chamber (Figure 6(a)). The temperature, humidity, and vacuum were kept constant in the testing chamber to prevent any unwanted performance variations due to temperature and humidity changes.

\section{Results and discussion}

\subsection{Fabricated devices}

The fabricated gyroscope is shown in Fig. 6(b). For the two-port resonator, the IDT finger pairs were five and the aperture length was $40 \lambda$. The cavity between the input and output IDTs was $50 \lambda$. The spacing between IDT and its adjacent shorted grating reflector was $5 \lambda / 8$. The 
number of metal stripes in the shorted grating reflector was 250. The SPUDT transducer was used for the delay lines. The finger pairs were 20 and the aperture length was set to $50 \lambda$. Three reflectors were positioned in a row. The metallic dots (number of dots: $49 \times 45$ ) were distributed on an anti-node positions of the standing wave between the two IDTs. The size of the metallic dots was approximately $12.4 \mu \mathrm{m} \times 11.4 \mu \mathrm{m}$.

\subsection{Wireless tests without rotation}

First, the two-port SAW resonator was tested by using a network analyzer. The frequencies were swept and the transmission coefficient $S_{21}$ was measured. As shown in Fig. 7, a $80 \mathrm{MHz}$ center frequency was observed, and the transmission peak was sharp and has narrow band width and low insertion loss. The insertion loss was approximately $-11 \mathrm{~dB}$. The experimental results were well matched with the predicted values from COM modeling. Wireless test was performed using antennas with double resonant frequencies. One antenna was connected to the network analyzer, and the other was connected to the fabricated gyrosensor. Electrical connection was completed as shown in Fig. 1. Two-port IDTs at the resonator and one-port IDT at the delay line were simultaneously linked with one antenna through the same ground port. Fig. 6(a) shows wireless testing setup and its results on the monitor in the network analyzer. Two $S_{11}$ peaks in the frequency domain were observed in a row under no rotation of the device (fig. 8) (In fig. 8, only the peak at $195 \mathrm{MHz}$ is shown in fig. 8, please fixe or refer to fig. 14). One is obtained from SAW resonator with $80 \mathrm{MHz}$ center frequency and the other was from SAW reflection delay line with $195 \mathrm{MHz}$ center frequency. Next, the obtained results in the frequency domain were converted to the time domain. A large $\mathrm{S} / \mathrm{N}$ ratio and clear reflection peaks were observed, as shown in Figure 8 . The three reflection peaks occurred at $0.6 \mu \mathrm{s}, 1.1$ $\mu \mathrm{s}$ and $1.3 \mu \mathrm{s}$, respectively. All the reflected peaks matched the predicted values from the COM modeling. 


\subsection{Wireless tests with rotation}

Next, the coriolis force effect was tested by revolving the fabricated devices. The sensor was placed on a rate table. The device was maintained under constant temperature, humidity, and vacuum conditions. The frequency was swept and the $S_{11}$ was measured at the network analyzer while rate table was rotated from $0 \sim 800 \mathrm{deg} / \mathrm{s}$. The reflection peaks were deviated from its reference point at the SAW reflective delay line because vibrating metallic dots at the antinode position of the standing wave caused a secondary wave, which interfered with the propagating SAW in the reflective delay line. As the revolution speed increased, the deviations at the second and third reflection peaks were linearly increased because a larger interference effect was enacted on the propagating SAW. The peak deviation in the time domain was transformed to values in the phase values following (phase $=\omega \cdot \Delta \tau$ ). Figure 9 shows the measured phase deviations of the SAW reflective delay line in terms of different rotation speeds $(0-800 \mathrm{deg} / \mathrm{s})$. The data points were calibrated to find the full scale range, linearity, and sensitivity. A good linearity was observed in wide range as shown in Fig. 9. The obtained sensitivity and its linearity were $1.35 \mathrm{deg} / \mathrm{deg} / \mathrm{s}$ and 0.91 , respectively. Those are competitive values with currently existing MEMS gyroscope. However, compared to mechanical gyroscopes using spinning wheel and optical fiber gyroscopes using Sagnac effects, it is still behind, but it is enough room for improvement in future by adjusting further parameter optimizations like operation frequency, device configurations, and by minimizing noise sources from measurement system.

Next, the device was rotated around different directions instead of around the standing wave propagation direction, which causes the Coriolis force to act to different directions. As shown in Fig. 9, very small phase deviations were observed when the devices were rotated around different directions instead of the standing wave direction. Good sensing directivity was 
observed. The coriolis force depends on the weight of metallic dot according to $F_{\text {coriolis }}=2 \cdot m \cdot(v \times \Omega)$. To check the mass effect, different masses of metallic dots on the top of the antinode position were deposited. As shown in Fig. 10, as the mass increases, the sensitivity was improved because a larger interference was acted by the secondary wave. However, heavy mass damps and crushes the standing wave, deteriorate the stably propagating standing wave, so that very carefulness is needed in case of choosing the weight of metallic dot $\mathrm{m}$.

\subsection{Thermal-related tests}

The effect of temperature on the device performance was tested. A change in temperature induces the elongation/contractions of the IDT finger periodic as well as it varies the SAW velocity and propagation length, so that it should be removed either by integrating a temperature sensor and then subtracting the temperature effect from whole gyro effect, or by using a compensation method within the sensor itself like a difference of method (DOM) [15]. To check a temperature effect, the temperature of the testing chamber was varied from 25 to 75 ${ }^{\circ} \mathrm{C}$ while the other parameters were kept constant during the testing period. Within a given test temperature range $\left(25 \sim 75^{\circ} \mathrm{C}\right)$, a relatively stable device performance was observed, as shown in Fig. 11. The substrate effects due to temperature variations was appropriately compensated using the methods mentioned above, but the coriolis force variation due to temperature effect is another problem to be resolved. Generally temperature variation causes change in the vibration velocity of the metallic dots located onto the antinode position on the standing wave, leading to a change in the magnitude of the coriolis force. Within a tested range of temperature, our gyroscope showed stable sensitivity and reliability, but further research for temperature compensation methods are needed upto extremely harsh environments for stable gyro use.

Next, additional heat generation was monitored along the surface with an infrared camera. The propagating SAW on the substrate generates a heat due to acoustic energy scatterings when 
the wave collides with lattices. This heat may cause an elongation of substrate and a decrease in the SAW velocity, resulting in a wavelength change of the SAW and the downshift of the center frequency [16-18]. When $1 \mathrm{~mW}$ RF power was applied, a small amount of heat was observed, whereas when the RF power increases, a sizable amount of heat was detected through the infrared camera, as shown in Figure 12(a,b). Red color in the image signifies a relative heat at those spots. As the power and the duration time of the power applied increase, more heat is observed from infrared camera. Based on these results, we suggest that a moderate RF input power should be applied to the IDTs. Applying a larger RF energy (e.g., more than $1 \mathrm{~W}$ ) is beneficial for inducing a wave with a large amplitude, but at the same time, it causes additional heat generations on the substrate and it should be removed. Pertinent RF power is demanded for activating the SAW devices.

\subsection{Impedance matching effect and other testing results}

Impedance matching circuit is needed because RF energy is applied to one port SAW delay line. For maximum power transfer, $\mathrm{R}=50 \Omega$ should be matched with impedance at the one port delay line. At the center (resonant) frequency, the susceptance component becomes to zero, but the static capacitance will be still remained, which should be cancelled out by parallel connection with corresponding inductor. By matching between $\mathrm{R}=50 \Omega$ and radiation impedance $\mathrm{R}_{\mathrm{IDT}}$, the impedance matching is obtained for maximum power transfer without any loss. For our case of the impedance matched circuits, the impedance passed through the center of the smith chart as shown in Fig. 13, showing a good impedance-matched design in our SAW delay line.

Currently our gyroscope can measure only one axis of angular velocity. But by placing a second gyroscope in an orthogonal position on the same substrate, two axis detectable gyro effects can be implemented at a time. Additionally, by placing a third gyroscope on the top of 
the device with a gap in vacuum through stacked configurations, 3 axis detectable SAW gyroscope is possible. To prevent any environmental effects, our sensor system was tested in a vacuum during all the testing periods. There is an energy loss when the SAW is surrounded by air during SAW propagation because of air loading and coupling. There is also another loss of SAW energy in air due to Brownian noise, which is caused by molecular collisions from the surrounding environment. These components reduce the amplitude of the SAW, as well as the resolution and the sensitivity. There are several methods for utilizing a vacuum package, such as the use of a commercially available vacuum packaging system by placing the gyrosensor into the package system, or hermetically sealing and encapsulating the device using a wafer bonding technique. In this test, we used the vacuum chamber to prevent any environmental effects. Major advantage of the SAW gyroscope over the gyroscopes using different phenomena is device stability under high shock and vibration environments because the SAW gyroscope does not have any suspended structures (proof-mass) to induce a Coriolis force, in contrast to MEMS gyroscopes. To prove the stability of devices under shock and vibration conditions, the device was shaken as shown in Fig. 14, and hammered down while measurement. Almost negligible changes in the device performance were observed until a shock of more than $15,000 \mathrm{~g}$ and a revolution of the device up to $800 \mathrm{deg} / \mathrm{s}$, proving that SAW gyroscope is very stable for shock and vibration.

\section{Conclusion}

In the present work, we have presented a novel wireless and battery-free SAW-based gyroscope utilizing a one-port reflective delay line and double resonant antenna. The optimal device structure was designed using the COM modeling. In the rate test, a Coriolis effect was observed with a vibrating metallic mass. The obtained sensitivity was approximately 1.35 $\mathrm{deg} /(\mathrm{deg} / \mathrm{s})$ at angular rate changes of $0-800 \mathrm{deg} /(\mathrm{deg} / \mathrm{s})$. From these results, we conclude that 
our wireless, battery-free SAW gyroscope is very promising for real-world smart system applications in which very high shock and vibration resistances are required.

\section{Acknowledgment}

This research was supported by the National Research Foundation of Korea (NRF) joint work project (2012033005). 


\section{References}

1. W. Wang, H. Oh, K. Lee, S. Yoon, and S. Yang: Japanese Journal of Applied Physics 48 (2009) 06FK9.

2. R. Woods, H. Kalami, and B. Johnson: IEEE Transactions on Ultrasonics, Ferroelectrics and Frequency Control 49 (2002 ) 136.

3. H. Oh, W. Wang, S. Yang, and K. Lee: Sensors and Actuators A: Physical 165 (2011) 8

4. W. Wang, J. Liu, M. Liu, and S. Yang: Applied Physics Express 4 (2011) 6601.

5. W. Wang, F. Xu, S. he, S. Li, and K. Lee: Japanese Journal of Applied Physics 49 (2010) 096602.

6. R. Woods, H. Kalami, and B. Johnson; IEEE Transactions on Ultrasonics, Ferroelectrics, and Frequency Control 49 (2002) 136.

7. K. Jose, W. Suh, P. Xavier, V. Varadan, and V. Varadan: Wave motion 36 (2002) 367.

8. V. Varadan, W. Suh, P. Xavier, K. Jose, and V. Varadan: Smart Materials and Structures 9 (2000) 898 .

9. M. Kurosawa, Y. Fukuda, M. Takasaki, and T. Higuchi: Sensors and Actuators A: Physical 66 (1998) 33.

10. S. Lee, J. Rhim, S. Park, and S. Yang: Journal of Micromechanics and Microengineering 17 (2007) 2272.

11. L. Reindl: Int. Symp. Acoustic Wave Devices for Further Mobile Communication Systems 2004, p. 1.

12. L. Reindl, A. Pohl, G. Scholl, and R. Weigel: IEEE Sens. J. 1 (2001) 69.

13. C. Campbell: Surface Acoustic Wave Devices for Mobile and Wireless Communications (Academic Press, London, 1998).

14. J. Gardner, V. Varadan, and O. Awadelkarim: Microsensors MEMS and Smart Devices (John Wiley and Sons, New York, 2001).

15. M. Jungwirth, H. Scherr, and R. Weigel: Acta Mechanica 158 (2002) 227.

16. T. Aubert, O. Elmazria, B. Assouar, L. Bouvot, and M. Oudich: Applied Physics Letters 96 (2010) 203503.

17. H. Oh, K. Lee, S. Yang, and K. Lee: Microelectronic Engineering 97 (2012) 259.

18. D. Lukyanov, Y. Filatov, S. Shevchenko, M. Shevelko, A. Peregudov, A. Kukaev, and D. Safronov: Gyroscopy and Navigation 2 (2011) 214. 
Fig. 1

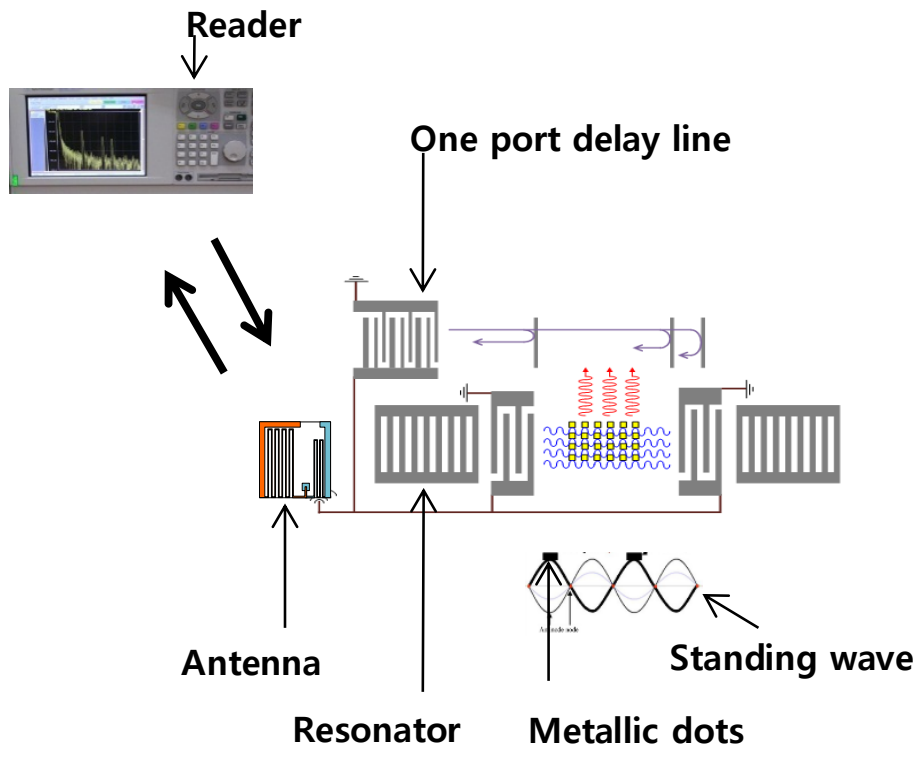

Figure 1. Schematic view of the developed gyrosensor system. It is composed of two SAW devices (two-port resonator and one-port reflective delay line), an antenna with double resonant frequencies $(80 \mathrm{MHz}$ and $195 \mathrm{MHz})$, and reader (network analyzer). The resonator produces constantly vibrating standing wave in which metal dots are positioned onto the antinode position of the standing wave.

Fig. 2

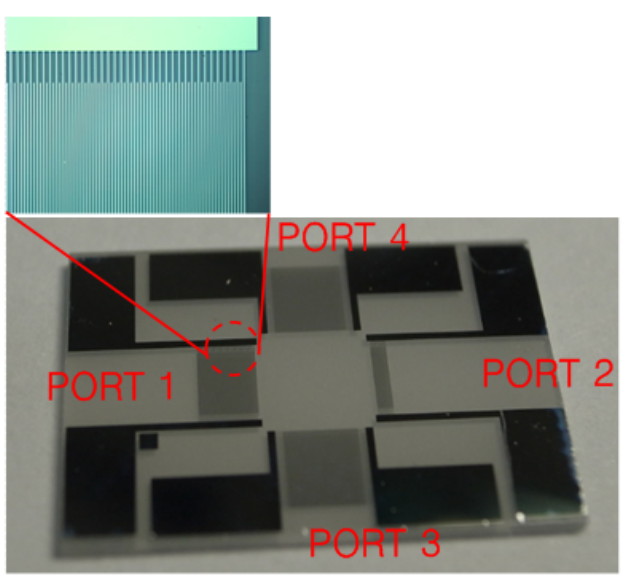

(a)

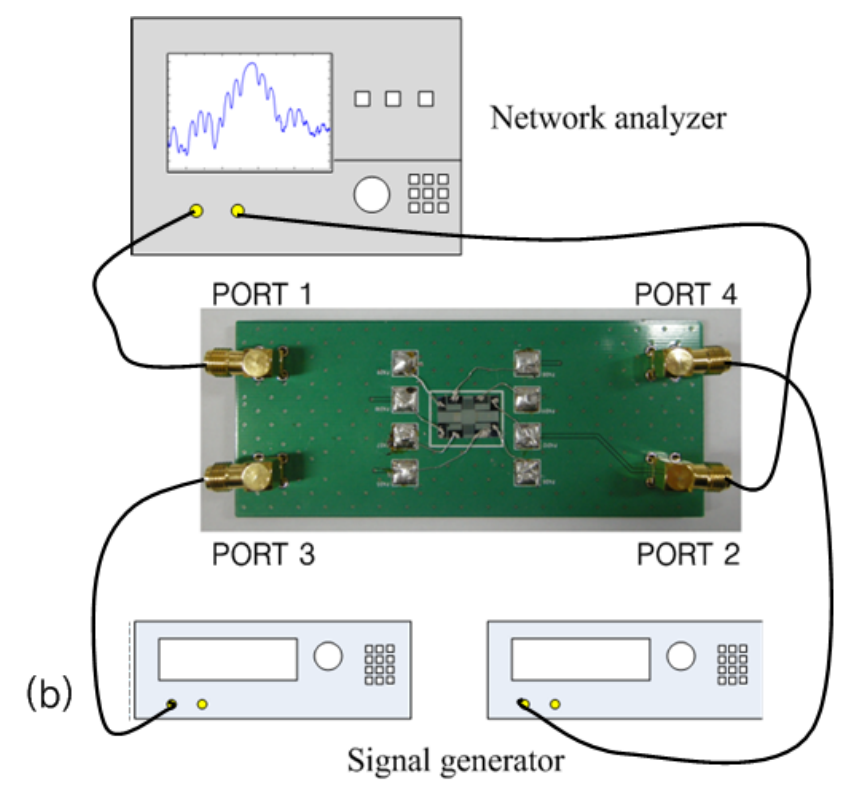

(b) 


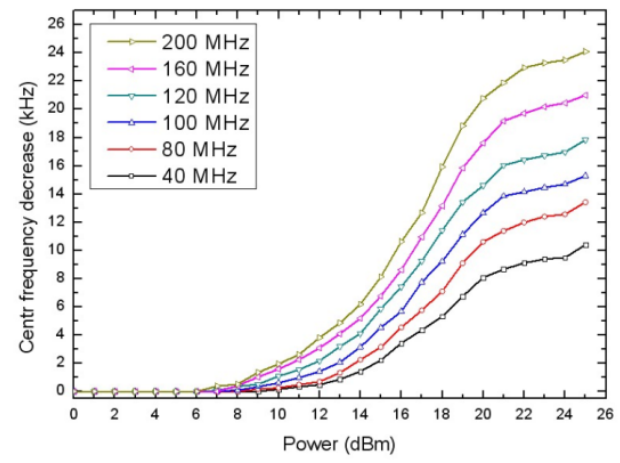

(c)

Figure 2. (a) Top view of the fabricated two SAW devices. SAW is propagating at the delay line, and at the same time, from the vertical side, another SAW with different center frequencies interferes the propagating SAW at the delay line. (b) Experimentally implemented configurations for testing. (c) As the input power and the center frequencies from vertical side become higher, the SAW propagating at the delay line becomes slower, and more downshifted at the center frequency in the transmission coefficient $\mathrm{S}_{21}$.

Fig. 3.

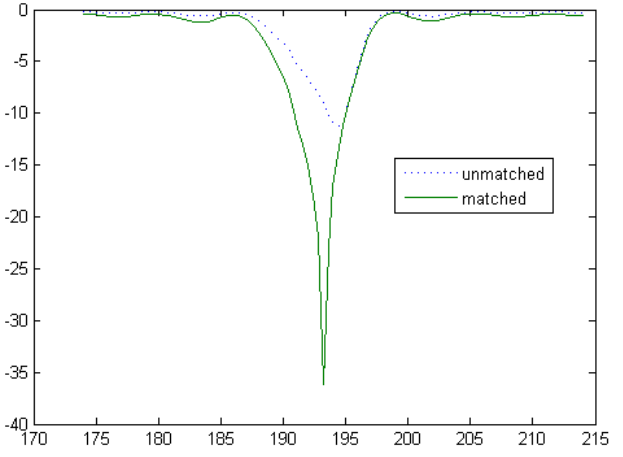

(a)

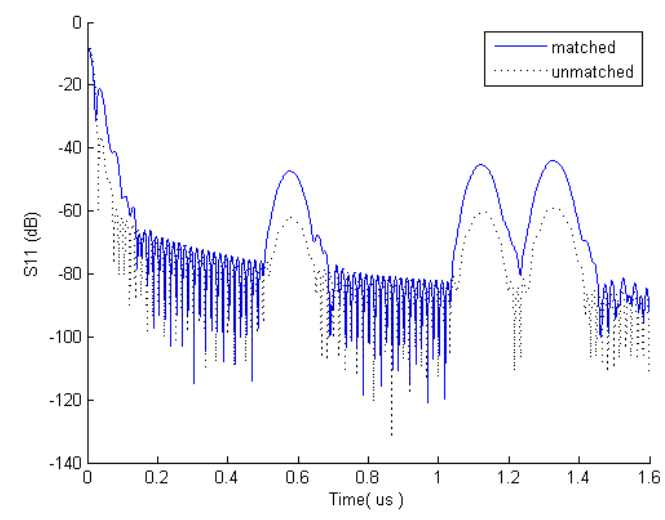

(b)

Figure 3. $\mathrm{S}_{11}$ responses obtained from one-port SAW reflective delay line in (a) frequency and (b) time domain. The center frequency was targeted to $195 \mathrm{MHz}$ and three reflectors with high $\mathrm{S} / \mathrm{N}$ ratio and uniform intensity were observed from impedance-matched delay line design. 
Fig. 4

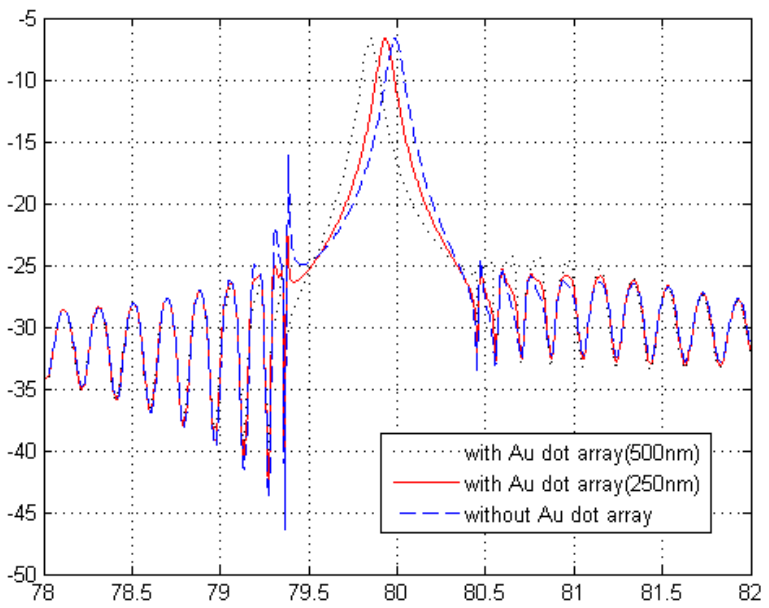

Figure 4. Two-port SAW resonator responses in terms of various metal dot array thicknesses. Heavy metal dots distort stable standing wave, causing a downshift in SAW velocity flowing underneath the metal dots and resonance frequency.

Fig. 5



(a) $128^{\circ} \mathrm{YX} \mathrm{LiNbO}_{3}$ substrate

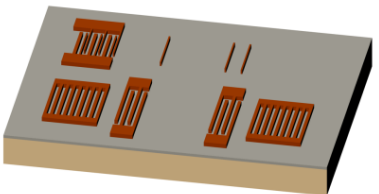

(c) PR coating \& patterning (AZ $1512)$

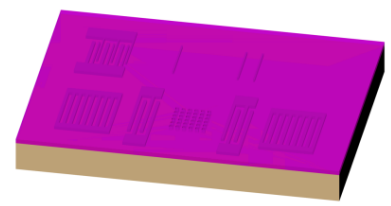

(e) PR pattern for lift-off(AZ 4620)

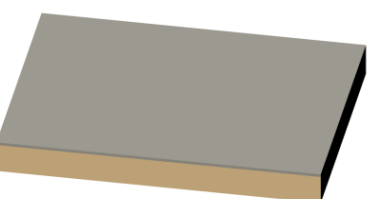

(b) Al deposition

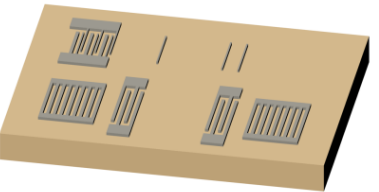

(d) Al etching \& remove PR



(f) Au dot array deposition by lift-off process

Figure 5. Fabrication procedure of the developed gyrosensor. 
Fig. 6



(a)

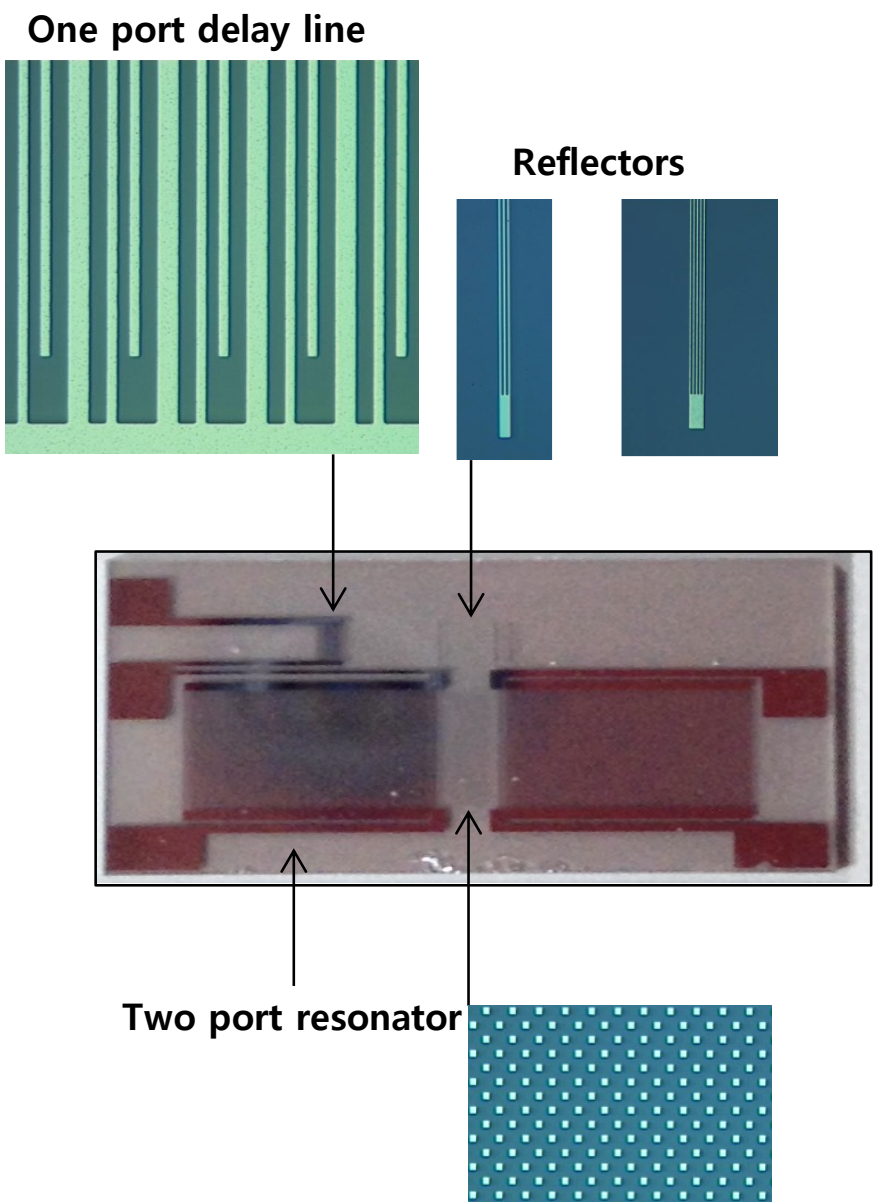

Metallic dots

(b)

Figure 6. (a) Measurement setup for wireless testing. Sensor was connected with antenna and mounted onto rate table. The temperature, humidity, and vacuum were kept constant in the testing chamber. (b) Entire view of the fabricated devices. 
Fig. 7

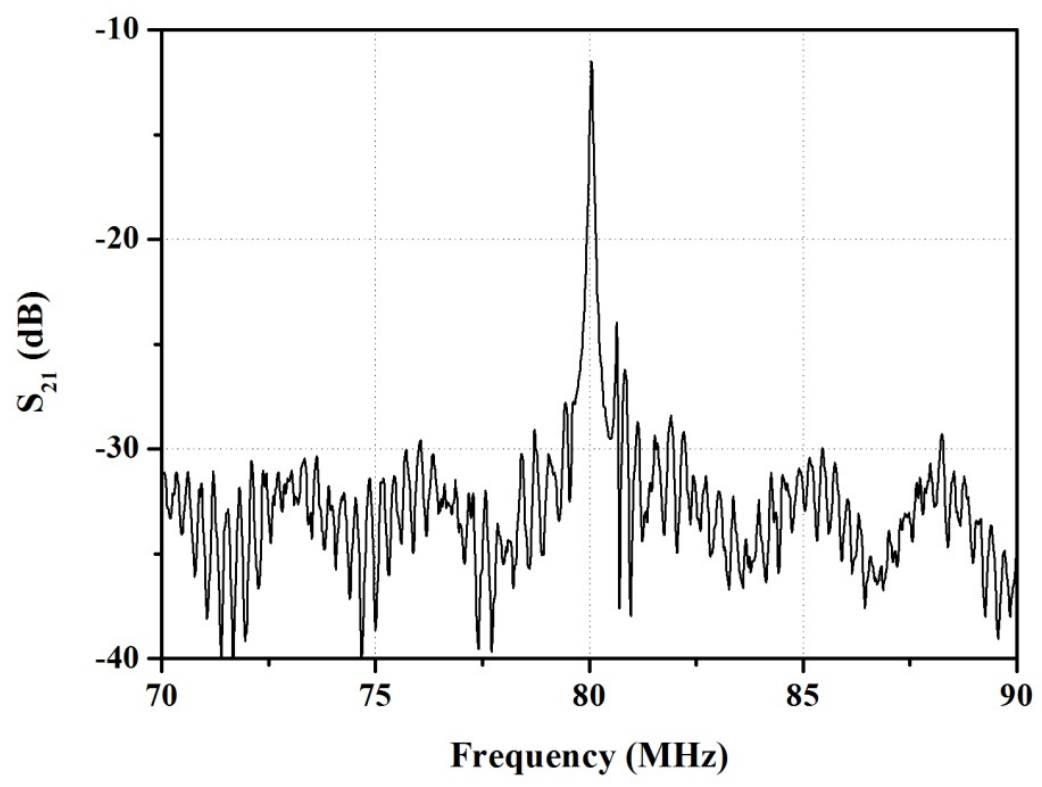

Figure 7. The transmission coefficient $S_{21}$ for two-port SAW resonator in terms of frequencies. The transmission coefficient was sharp and has narrow band width. 
Fig. 8



(a)

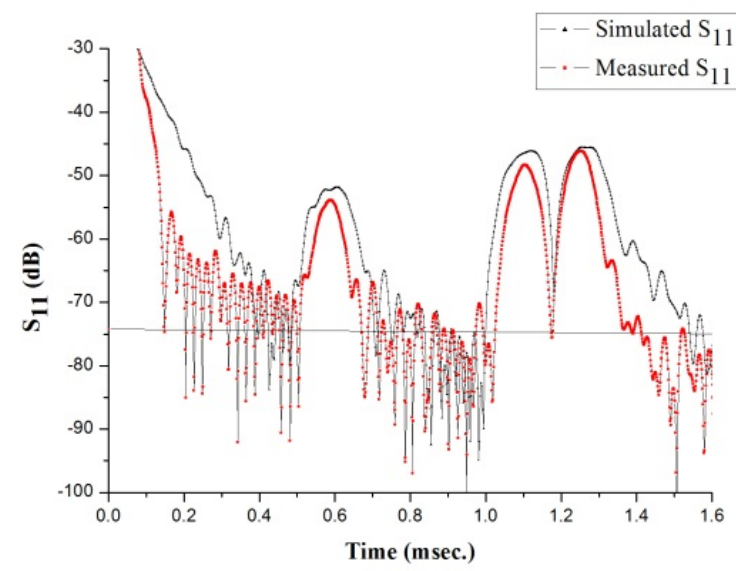

(b)

Figure 8. Reflection peaks $\left(\mathrm{S}_{11}\right)$ of one-port SAW reflective delay line in (a) frequency domain and (b) time domain in which peaks were well matched with the predicted values from simulation.

By comparison to Fig. 3-b, I think that the simulated S11 is the red curve and not the black one ?? 
Fig. 9

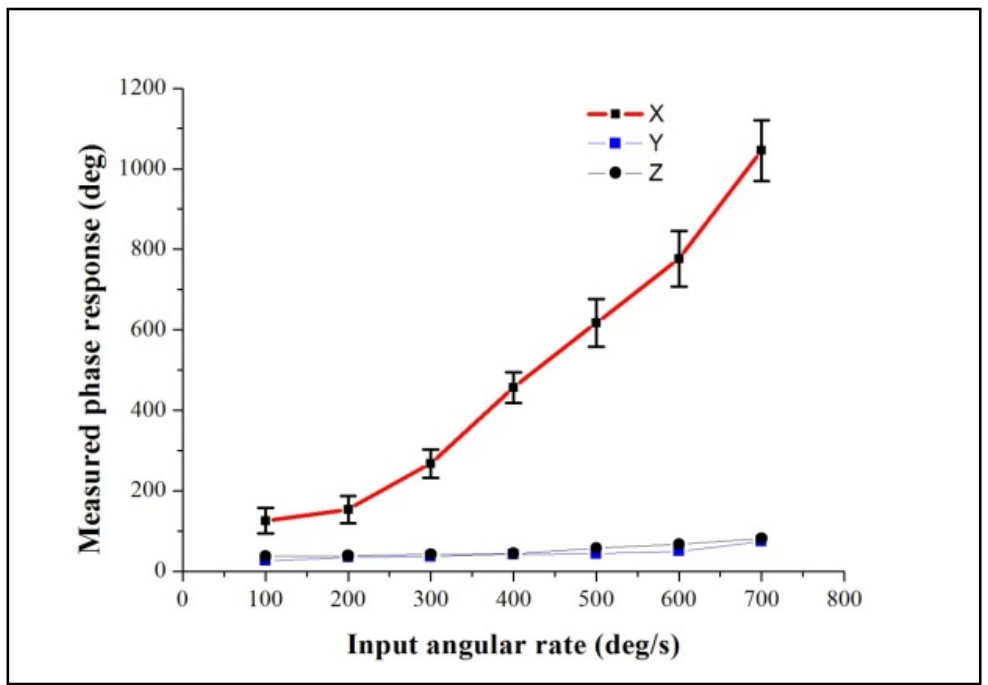

Figure 9. Measured phase responses from one-port reflective delay line when device was revolved around the standing wave propagation direction and comparison of the phase responses when the device was revolved about different directions

Fig. 10

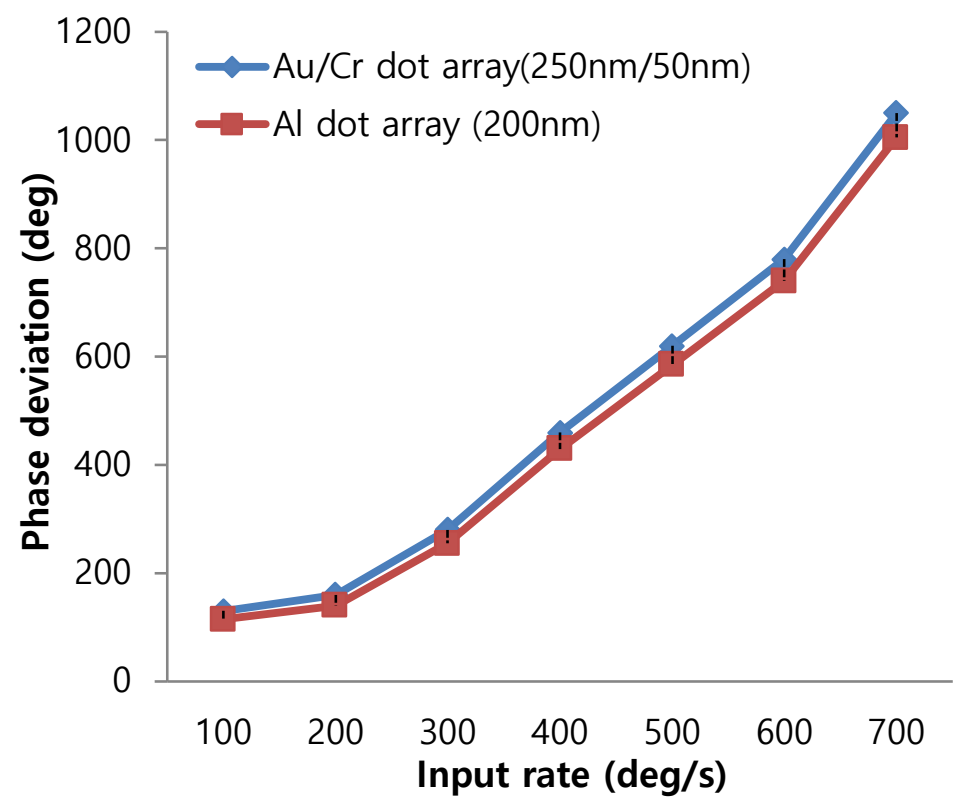

Figure 10. Measured phase responses from one-port reflective delay line in terms of different masses of metallic dot array when device was revolved around the standing wave propagation direction. Heavier mass of array shows better sensitivity. 
Fig. 11



Figure 11. Measured phase responses from one-port reflective delay line in terms of different temperatures when device was revolved around the standing wave propagation direction.

Fig. 12



(a)

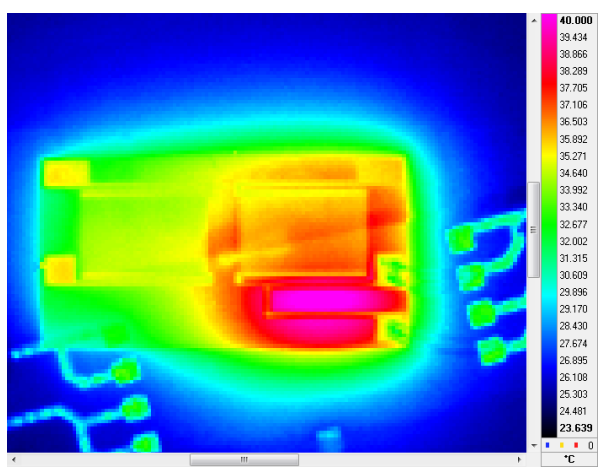

(b)

Figure 12. Infrared testing setup for heat generation and spreading effects during SAW propagation (a) when $1 \mathrm{~W}$ RF power was applied to one-port SAW delay line, and (b) as the power and the duration time of the power increase, more heat is observed. 
Fig 13

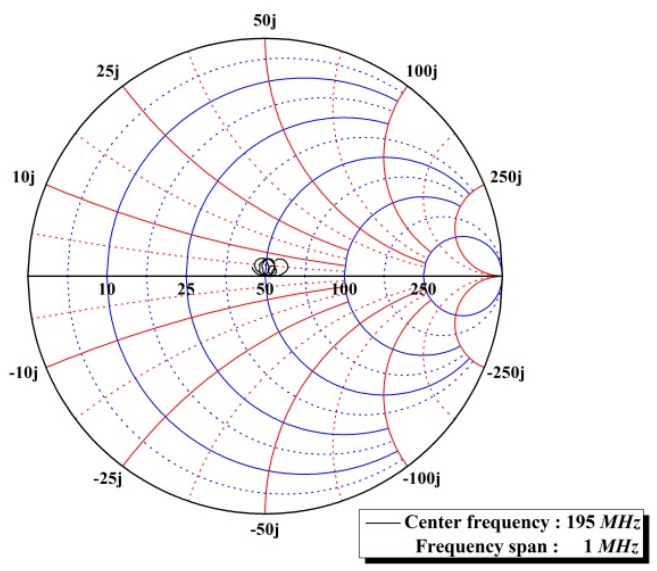

Figure 13. Smith chart showing impedance matching of our device nearby $195 \mathrm{MHz}$ center frequency in one-port delay line. The impedance passes through the center of the smith chart nearby $195 \mathrm{MHz}$ center frequency.

Fig. 14

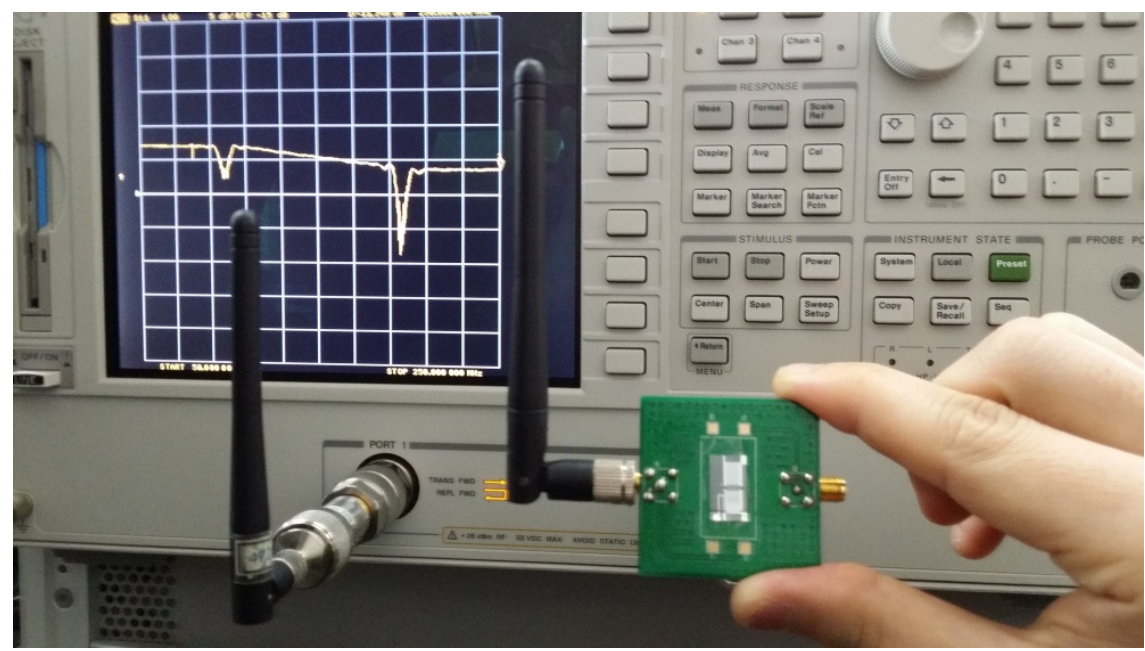

Figure 14. Device was shaken and wirelessly measured in network analyzer. No noticeable perturbation in performances was not observed, proving this device is very stable for vibration. 